\title{
Editorial: Probleme des Klassenkampfs - heute
}

A

m 19. Juni 2020, dem »Tag der Sklavenbefreiung«, bestreikten die Mitglieder der Hafenarbeitergewerkschaft ILWU 29 Häfen an der US-Westküste. Nahezu nichts ging mehr. Die Arbeiter und Arbeiterinnen solidarisierten sich mit den anhaltenden Black-Lives-MatterProtesten, die das ganze Land nach dem Mord an George Floyd in Atem hielten. Aus New York, jener Stadt in den USA, die am schwersten von der CoronaPandemie erfasst wurde, waren Bilder zu sehen, wie Krankenhaus- und Pflegepersonal kurz vor Dienstantritt, bereits in voller Schutzmontur, auf der Straße den Protesten applaudierten. Von vielen Protestierenden schallte ein von Masken gedämpftes »Thank you!« zurück. Ähnliche Szenen waren auch vor anderen Krankhäusern zu sehen.

Beispiele wie diese zeigen, dass die Black-Lives-Matter-Proteste auf vielfältige Weise mit Kämpfen in der Arbeitswelt verbunden sind, zumindest über symbolische Solidaritätsbekundungen. Doch für uns als eine Zeitschrift, die vor fast fünfzigJahren erstmals unter dem Titel Probleme des Klassenkampfs und bereits seit 1976 unter dem Akronym PROKLA erscheint, stellt sich die Frage, was die aktuell wirkmächtigsten sozialen Bewegungen und Kämpfe wie Black Lives Matter, aber auch Fridays for Future oder Mietenproteste jenseits dieser symboli- schen Solidaritätsbekundungen grundsätzlich mit Klassenkämpfen zu tun haben. Zwar wird in Deutschland spätestens seit dem Erscheinen von Didier Eribons Rückkehr nach Reims (2016) - teilweise heftig - über das Verhältnis von Identitäts- und Klassenpolitik vor dem Hintergrund des Aufstiegs der Rechten und der Krise der Sozialdemokratie diskutiert. Zugleich aber scheinen sich die treibenden emanzipatorischen Kräfte und Bewegungen gesellschaftlicher Veränderung nicht erst in jüngster Zeit in vielen Fällen jenseits von Klassenverhältnissen und -kämpfen zu entzünden. Ist die Kategorie Klassenkampf also viel zu grob, wenn nicht gar obsolet, um die Dynamik heutiger Gesellschaften zu verstehen? Leistet der Fokus auf die Kategorie des Klassenkampfs möglicherweise sogar einer »Rückkehr zum Hauptwiderspruch» Vorschub, vor dem Emma Dowling, Silke van Dyk und Stefanie Graefe (2017) warnen? Kurz: Warum heute noch eine Zeitschrift in der Tradition machen, die den Klassenkampf und seine Probleme ins Zentrum stellt?

Vor fast fünfzig Jahren, im November 1971, erschien das erste Heft der Probleme des Klassenkampfs mit dem Untertitel Zeitschrift für politische Ökonomie und sozialistische Politik. Die Welt erschien damals zwar in großer Unordnung, aber begrifflich war sie aus 
linker Sicht relativ einfach zu ordnen. Es war klar, auf welcher Seite man zu stehen hatte. Man war (unter anderem) gegen die USA im Vietnamkrieg, gegen die südafrikanische Regierung und ihre Politik der Apartheid, gegen die Militärdiktatur in Brasilien und für Salvador Allende in Chile sowie für die antikolonialen Bewegungen auf dem afrikanischen Kontinent. Was die Bundesrepublik selbst betraf, war die Linke zwar zutiefst uneins über die Rolle des Staates, die richtige revolutionäre Taktik und den realen Sozialismus in der UdSSR oder in der Volksrepublik China. Dagegen gab es eine allgemeine Übereinstimmung darüber, dass im Zentrum aller sozialen Auseinandersetzungen die Arbeiterklasse stand.

Im Editorial der ersten Ausgabe benannten die 23 Gründungsmitglieder der neuen Zeitschrift die konkrete Analyse kapitalistischer Gesellschaften als ihr zentrales Anliegen. Sie lehnten es ab, der Zeitschrift »einen weitgespannten theoretischen Programmentwurf " bestehend »aus Allgemeinheiten und abstrakten Postulaten « vorauszuschicken. Die theoretische und politische Linie werde "sich vielmehr in den in der Zeitschrift vorzulegenden Analysen im einzelnen, d.h. am Gegenstand, darstellen und konkretisieren müssen." (PROKLA-Redaktion 1971: 1) Damit grenzten sich die Gründungsmitglieder zum einen von der Sozialistischen Politik (SoPo) ab, also jenem ZeitschriftenProjekt, dessen Usurpation durch ein DKP/SEW-nahes Redaktionskollektiv den Anlass zur Gründung der Probleme des Klassenkampfs gegeben hatte. Zum anderen wandten sie sich gegen die dogmatischen Verhärtungen und abs- trakten Bekenntnisse zum »Standpunkt des Proletariats«, wie sie für viele der aus der Studierendenbewegung hervorgegangenen Kleinstparteien und Organisationen kennzeichnend war. David Bebnowski rekonstruiert aus Anlass des Heftjubiläums diese Anfangsjahre der PROKLA. Er beleuchtet die Entstehungsgeschichte unserer Zeitschrift im Verlauf der 1960er und 1970er Jahre und zeigt, dass sie durch eine eigene theoretische Positionierung innerhalb des marxistischen Denkens und durch ihre Abgrenzung zu doktrinären Positionen ihren bleibenden Charakter gewann.

Der Klassenkampf war der zentrale Bezugspunkt auch der neuen Zeitschrift. Ihn durch die wissenschaftliche "Analyse und Kritik der bürgerlichen Gesellschaft « zu befördern, war das politische Projekt der Gründungsmitglieder und gleichzeitig die "naheliegendste [...] Aufgabe marxistischer Intelligenz « (ebd.: 4), deren Erfüllung einen organischen Zusammenhang zwischen linken Intellektuellen und den gesellschaftlichen Kämpfen der damaligen Zeit herstellen würde.

Doch bereits zum Zeitpunkt der Gründung von Probleme des Klassenkampfs und dann verstärkt im weiteren Verlauf der 1970er und 1980er Jahre drängten andere Konflikte in den Vordergrund. Aus der Studierendenbewegung erwuchsen nicht nur die K-Gruppen, sondern auch eine große Vielfalt neuer Ansätze und Bewegungen, die sich etwa Problemen der (damals sogenannten) Dritten Welt, der gesellschaftlichen Lage von Frauen, der Umwelt oder des Weltfriedens angesichts der atomaren Bedrohung widmeten und deren Vorgeschichte teilweise bis in die 1950er 
Jahre zurückreichte. Als Auslöser der Umweltbewegung gilt beispielsweise das 1962 erschienene und später zum Klassiker avancierte Buch von Rachel Carson Der stumme Frühling, das einer breiten Öffentlichkeit erstmals die verheerenden Wirkungen von Pestiziden in der Landwirtschaft vor Augen führte. Die Kämpfe der Lohnabhängigen blieben davon nicht unberührt. So sind die betrieblichen Ansätze zur Konversion der Rüstungs- und der Automobilindustrie der 1970er und 1980er Jahre auch im Kontext eines von der Friedens-, der Ökologie- und der Internationalismusbewegung beeinflussten gesellschaftlichen Klimas zu sehen.

Die noch dem Editorial des ersten Hefts der Zeitschrift zugrunde liegende Annahme, man befinde sich einer Phase "sich verschärfender Klassenkämpfe« (PROKLA-Redaktion 1971: 3) erwies sich hingegen als unzutreffend - zumindest, wenn der Begriff des Klassenkampfs eine offene Auseinandersetzung zwischen Kapital und Arbeit definiert. Noch 1952 hatte es in der neuen Bundesrepublik intensive Auseinandersetzungen um das Betriebsverfassungsgesetz gegeben. Den Gewerkschaften, die die Montanmitbestimmung auf die gesamte Wirtschaft ausdehnen wollten, war es gelungen, mehr als eine Million Arbeiter und Arbeiterinnen zu mobilisieren. 1952 beschloss die bürgerliche Mehrheit im Bundestag allerdings ein Betriebsverfassungsgesetz, in dem die zuvor formulierten Vorstellungen und erhobenen Forderungen mitnichten berücksichtigt wurden - und das obwohl Streiks und Klassenkämpfe die Jahre zuvor in den Westzonen prägten und es eine breite Debatte um Sozialisierung sowie Wirtschaftsdemokratie gab. In den folgenden Jahren kam es jedoch auch zu erfolgreichen Streiks für kürzere Arbeitszeiten, höhere Löhne und die Lohnfortzahlung im Krankheitsfall. Besonders in der Druckindustrie zeigten sich die Arbeiter*innen streikfreudig, ebenso die Metaller*innen. Diese traten 1969 spontan gegen Tarifverträge mit langen Laufzeiten, die sie zur Immobilität verdammten, in den Ausstand - erstmals im Gegensatz zur Politik ihrer Funktionäre. Auch das gewerkschaftliche Projekt Humanisierung des Arbeitslebens fand breite Unterstützung bei den Beschäftigten und man suchte ihm mit Arbeitskampfmaßnahmen Nachdruck zu verleihen. Dagegen führte die erste große Nachkriegs-Krise von 1975 zwar zu einem sprunghaften Anstieg der Arbeitslosigkeit, nicht jedoch zu der von Linken erwarteten Radikalisierung von Arbeitskämpfen. Das innerhalb der Probleme des Klassenkampfs bestehende Redaktionskollektiv Gewerkschaften stellte dazu fest, das Ausbleiben einer politischen Antwort seitens der Arbeiterklasse scheine »den von Marx postulierten Zusammenhang von ökonomischer und Klassenbewegung zu widerlegen. Denn schließlich kann keine Rede davon sein, daß die Erfahrung des antagonistischen Interessengegensatzes die westdeutschen Lohnabhängigen in den Klassenkampf gegen das Kapital gegenwärtig zwingt, vielmehr stößt man allenthalben auf die Ansicht, Resignation, privater Rückzug und die Aufgabe fortschrittlicher Positionen seien das wesentliche Resultat der kapitalistischen Krise. Fortschrittlich-politische Perspektiven, die in der beginnenden Ära der sozialliberalen 
Koalition entstanden seien, gingen zunehmend zugunsten einer Reduktion auf reine Interessenwahrnehmung verloren. (Redaktionskollektiv Gewerkschaften 1975: 3) Die Klassenkämpfe, die der Zeitschrift bis dahin ihren Namen gegeben hatten, wurden ab 1976 von der Redaktion zum Akronym PROKLA verkürzt, der Untertitel Zeitschrift für politische Ökonomie und sozialistische Politik blieb zunächst erhalten, wurde dann aber 1992 in Zeitschrift für kritische Sozialwissenschaft geändert.

Das Umfeld für betriebliche Klassenkämpfe hatte sich seit den 1970er Jahren offenbar deutlich verschlechtert. Der gewerkschaftliche Organisationsgrad lag 1950 bei 34 Prozent und hielt sich auch 1980 noch bei 32 Prozent. Seither ging er stetig zurück. In der öffentlichen Wahrnehmung galten Gewerkschaften bis Mitte der 1980er Jahre als gesellschaftlicher Stabilitätsfaktor, trotz aller rabiaten Klassenkampf-Rhetorik der Arbeitgeberverbände. Doch dann drehte sich der Wind: Eine neue, mit der Verschiebung von Kräfteverhältnissen verbundene Marktgläubigkeit, gewann in der breiten Öffentlichkeit zunehmend Raum, der "Standort Deutschland « wurde als gefährdet inszeniert und Gewerkschaften galten mehr und mehr als "Dinosaurier der Industriegesellschaft«, als Bremser gegen jeglichen Wandel und die erforderliche Anpassung an die neuen Verhältnisse einer zunehmend globalisierten Wirtschaft. Der gesellschaftliche Bezugspunkt marxistischer Analyse drohte damit verloren zu gehen, der organische Zusammenhang zwischen kritischer Wissenschaft und Klassenkämpfen war in Auflösung begriffen. Im Editorial von PROKLA-Heft
43, das bezeichnenderweise unter dem Titel »Probleme mit dem Klassenkampf« erschien, wurde dies treffend diagnostiziert: Zwar sei nicht zu bezweifeln, "daß Analysen der gegenwärtigen gesellschaftlichen Verhältnisse auf der Grundlage der Marxistischen Theorie - bei allen empirischen, theoretischen und methodischen Schwierigkeiten - prinzipiell zur Aufklärung über die Gesellschaftsbedingungen, unter denen praktisches Handeln stattfindet, unerläßlich sind. In Zweifel geraten ist jedoch, in welchem Sinn und für wen diese marxistischen Analysen emanzipatorisch handlungsorientierend sind, d.h. in welchem Sinn sie eine sozialistische Praxis begründen. «(PROKLA-Redaktion 1981:2) Diese Zweifel wurden auch durch den Aufstieg der neuen sozialen Bewegungen genährt, der sich parallel zur Verschiebung der Kräfteverhältnisse zwischen Arbeit und Kapital vollzog. Vor allem in der Ökologiebewegung schienen das Gattungsinteresse und die Kritik am Industrialismus den Klassenkampf und die Kapitalismuskritik in den Hintergrund zu drängen. Gerade in der PROKLA blieb das jedoch nicht unwidersprochen. Schon in Heft 39 (1980) hatte sich die Redaktion mit dem Verhältnis von Arbeiter*innen- und ökologiebewegung beschäftigt und darauf hingewiesen, dass die Ausbeutung von Natur und Arbeitskraft einen gemeinsamen Ursprung in der Kapitalverwertung habe (PROKLA-Redaktion 1980:1f.).

Die Probleme nicht nur mit dem Klassenkampf, sondern auch mit dem Klassenbegriff blieben jedoch. Mehr noch: Sie verschärften sich. In PROKLA-Heft 100 (1995) diagnostizierte Bodo Zeuner unter dem Titel »Probleme des Klas- 
senkampfs $<$ im Betrieb «, dass ungeachtet des titelgebenden Akronyms weder Klassen noch Klassenkämpfe in der PROKLA (und anderswo) noch eine größere Rolle spielten. Der letzte Artikel dazu sei in den späten 1980er Jahren erschienen: »Der Klassenbegriff versickert fast unbemerkt aus dem linken sozialwissenschaftlichen Diskurs."(Zeuner 1995: 342) Dabei verortete er beim marxistischen Klassenbegriff drei wichtige Dimensionen zur Analyse der damaligen Debatten und ihres oftmals widersprüchlichen Bezugs auf reale Entwicklungen: Erstens stelle sich die Frage, inwiefern der Klassenbegriff in der marxschen Tradition überhaupt noch brauchbar sei, um die Struktur kapitalistischer Gesellschaften zu erklären. Zweitens ergäben sich daraus die weiteren Fragen nach der Beziehung zwischen Klassenlage und Klassenhandeln, und ob sich die Lücke zwischen Lage und Handeln zunehmend vergrößere. Dies führe drittens dazu, dass die an Marx anschließende Konflikttheorie, die den Klassenkampf ins Zentrum stelle, »für entwickelte kapitalistische Gesellschaften angesichts der zunehmenden sozialen und politischen Relevanz nicht darauf reduzierbarer Spaltungslinien « infrage gestellt werde (ebd.: 350).

Bei der ersten Frage nach der Brauchbarkeit des Klassenbegriffs bildet der Begriff der Mittelklasse den Ausgangspunkt einer Reflexion, wie sie in der PROKLA bereits in den 1980er Jahren (Wright 1985) sowie verstärkt in jüngerer Zeit angestellt worden ist (vgl. Milios/Economakis 2014; Kadritzke 2016; Thien 2014; Sablowski/Thien 2018). Die durchaus unterschiedlichen Positionen und Klassifizierungen waren und sind von dem gemeinsamen Bestreben motiviert, den Veränderungen in der Klassenstruktur kapitalistischer Gesellschaften Rechnung zu tragen, ohne den Klassenbegriff selbst zu vernachlässigen, wie dies lange Zeit in anderen sozialwissenschaftlichen Zeitdiagnosen (etwa der der Individualisierung) zu beobachten war. Mittlerweile ist der Klassenbegriff zwar auch jenseits des marxistischen Denkens zurück in der sozialwissenschaftlichen Debatte (Reckwitz 2019), was nicht zuletzt an der verbreiteten Diagnose des Verschwindens der sogenannten Mittelklassen innerhalb von "Abstiegsgesellschaften" (Nachtwey 2016) liegt. Allerdings bleibt er dabei entweder unscharf oder wird gar im Sinne von Schichten, Milieus oder »Kulturklassen « verwendet. An derartige Vorstellungen haben auch Ansätze angeschlossen, die eine neue Konfliktachse zwischen Kommunitarist*innen und Kosmopolit*innen oder den somewheres und den anywheres ausmachen (Goodhart 2017; Merkel 2017; kritisch: Graf 2019). Als ein der Kritik der politischen Ökonomie verpflichtetes Projekt will die PROKLA hier genauer hinschauen. Weder sollten Strategien der »Entsorgung " greifen können, noch sollten die Erkenntnisse der letzten Jahrzehnte verloren gehen. Stattdessen geht es darum, die Einsichten einer in der marxschen Tradition stehenden und mit anderen Strömungen kritischen Denkens verbundenen Klassentheorie für die Analyse aktueller gesellschaftlicher Entwicklungen fruchtbar zu machen.

Dies gilt auch für die zweite 1995 von Zeuner aufgeworfene Frage, nämlich die nach dem Zusammenhang von Klassenlage und Klassenhandeln. Die Men- 
schen machen ihre eigene Geschichte, aber nicht aus freien Stücken, nicht unter selbstgewählten, sondern unter unmittelbar vorgefundenen, gegebenen und überlieferten Umständen - so Marx' berühmte Formulierung im Achtzehnten Brumaire des Louis Bonaparte. Dabei wird seit Jahrzehnten darüber gestritten, wie die Umstände genauer zu bestimmen sind, wie sie als »Alp auf dem Gehirne der Lebenden " wirken, und was es bedeutet - sowie was es aus emanzipatorischer Perspektive dazu bedarf -, die Geschichte selbst zu machen. Die Antworten sind so vielfältig wie die Fragestellung nahezu immer ähnlich, in der Soziologie meist um einen gehaltvollen Klassenbegriff erleichtert, als Frage nach dem Verhältnis von Struktur und Handlung. Versteht man den Begriff der Klasse als einen, der Struktur und Handlung verbindet, dann stellt sich die Frage, unter welchen Umständen es $\mathrm{zu}$ welcher Form von Klassenhandeln kommt. Zeuner argumentiert, dass »keinerlei Determinismus [...] entlang der Ableitungslinie Klassenlage-Klassenbewußtsein-Klassenkampf [ [...] anzunehmen « (Zeuner 1995: 342) sei, sondern, dass das Klassenhandeln selbst als ein "politische[r] Prozeß« (ebd.) zu begreifen ist. In ähnlicher Weise begreift Alex Demirović in seinem Beitrag in diesem Heft Klassenkämpfe als Konfliktdynamik, die allen gesellschaftlichen Praktiken zugrunde liegt. Klassenkämpfe finden damit nicht nur dann statt, wenn sie sich explizit als solche artikulieren und organisieren, sondern bestimmen stets das Zusammenleben der Menschen. Klasse beinhaltet dabei immer den Kampf. Dieser ist der Existenz von Klassen nicht nachgelagert.
Die dritte von Zeuner aufgeworfene Frage ist die nach der Zentralität des Klassenkonflikts und nach dem Verhältnis zwischen letzterem und anderen gesellschaftlichen Widersprüchen. Diese Frage steht spätestens seit dem Aufkommen der neuen sozialen Bewegungen auf der Tagesordnung linker Politik und kritischer Sozialwissenschaft. Aktuell gewinnt sie zudem angesichts "demobilisierter Klassengesellschaften « an Bedeutung, in denen "Bewegungen und Organisationen, die dem Klassenkampf von oben durch Mobilisierung eigener Machtressourcen etwas entgegensetzen können" (Dörre 2019: 22), an Einfluss verloren haben. Nicht allein die Klassenposition, sondern eben auch die Position in anderen Herrschaftsverhältnissen zählt: Zentrale Konflikte der Gegenwart entzünden sich an Geschlechterverhältnissen, an rassistischen Verhältnissen und an den gesellschaftlichen Naturverhältnissen. Darüber hinaus ergibt sich die Klassenposition nicht mehr allein aus dem »Betrieb《 als Organisationsform des unmittelbaren Produktionsprozesses. Der »Betrieb « unterliegt seit Jahren einem permanenten Veränderungsprozess. Das macht den betrieblichen Kampf nicht nur schwerer, sondern sorgt auch dafür, dass Klassenkonflikte sich in andere Felder des Lebens verschoben haben, auch wenn sie sich nicht unmittelbar als solche artikulieren: Mietund Wohnverhältnisse, die öffentliche Daseinsvorsorge und Reproduktionskämpfe, kulturelle Fragen, Kämpfe um den öffentlichen Raum, um Bildung aber auch für "Solidarität « in der Euro-Krise oder während der Corona-Pandemie haben häufig einen Klassencharakter (vgl. den Beitrag von Alex Demirović in 
diesem Heft sowie die Debatte um eine neue bzw. »verbindende« Klassenpolitik in Friedrich 2018; LuXemburg 2017; Riexinger 2018).

Lässt man die vielen Schwerpunkte der letzten Jahre Revue passieren, so hat die PROKLA diesen Formen der (Klassen-)Konflikte viel Aufmerksamkeit zukommen lassen. Heft 200 haben wir nun zum Anlass genommen, die wissenschaftliche und politische Relevanz des Klassenkampf-Begriffs nochmal expliziter in Augenschein zu nehmen, um die "Probleme des Klassenkampfs heute" besser zu begreifen. Angesichts sich häufender und verschärfender Krisen stellen sich viele der in früheren Heften und natürlich auch in anderen Kontexten aufgeworfenen Fragen in einer neuen - vermutlich seit Bestehen der Zeitschrift nie dagewesenen - Dringlichkeit: Wo beginnen Klassenkämpfe, welche gesellschaftlichen Auseinandersetzungen und Praktiken sind darunter zu zählen? Wie gestaltet sich das Verhältnis von Klassenlage, Klassenerfahrung und Klassenkampf? Inwieweit handelt es sich bei den vielfältigen Konflikten, die in den vergangenen Jahren im globalen Süden und im globalen Norden aufgeflammt sind, um Klassenkämpfe? Worin liegt der klassenpolitische Gehalt antirassistischer, feministischer und ökologischer Kämpfe? Warum ist es wichtig, diesem Gehalt nachzuspüren, inwieweit lassen sich historische und zeitgenössische Auseinandersetzungen dadurch besser begreifen? Welchen Beitrag zur Orientierung emanzipatorischer Konflikte kann die Arbeit mit dem Klassenkampf-Begriff leisten?

Eine These, die sich wie ein roter Faden durch unser Heft zieht, besagt, dass Konflikten, die früher als Nebenwidersprüche abgetan wurden, durchaus eine Klassendimension zukommt. Damit hat sich auch das Feld dessen ausdehnt, was wir heute als politökonomisch orientierte Zeitschrift unter den "Problemen der Klassenkämpfe» (im Plural) fassen müssen. Dies zeigen Jakob Grafund Anna Landherr in ihrem Beitrag zu Klassenkämpfen im globalen Süden anhand des chilenischen Falles. Dort verbinden sich verschiedene Konfliktlinien zu einem breiten gesellschaftlichen Kampf gegen die »besitzende Klasse« des Landes. Klassenkämpfe dieser Art sind jedoch durch spezifische Austragungsformen, Subjekte und Machtressourcen gekennzeichnet. Markus Wissen geht dem Verhältnis von ökologischen Konflikten und Klassenkonflikten nach. Obwohl beide in derselben Gleichgültigkeit der kapitalistischen Produktionsweise gegenüber ihren eigenen Voraussetzungen gründen, können sie auseinanderfallen oder sich sogar gegeneinander wenden. Historisch war dies vor allem im Fordismus der Fall. Mit dem BrüchigWerden fordistischer Produktions- und Konsumnormen ergibt sich die Chance, ökologische und Klassenkämpfe in der Gebrauchswertorientierung (wieder) zusammenzuführen. Dorothea Schmidt blickt auf zwei Revolutionen im 20. Jahrhundert zurück. In ihrem vergleichenden, sozialgeschichtlich angelegten Beitrag zur russischen Revolution 1917 und zur deutschen Revolution 1918 analysiert sie die dahinterliegenden Klassenkonflikte. Dabei stellt sie die Frage in den Mittelpunkt, inwieweit es sich in Russland tatsächlich um Klassenauseinandersetzungen handelte und wie diese im Vergleich zu den Entwicklungen im 
Deutschen Kaiserreich zu sehen sind, das ein Jahr nach der russischen Oktoberrevolution ebenfalls zu Bruch ging. Ihr Augenmerkt liegt bei der Bedeutung, die den jeweiligen Klassenkämpfen vor dem Hintergrund der entstandenen Räte bei der Durchsetzung der neuen politischen Ordnungen zukamen. Thomas Sablowski analysiert die Wirtschaftspolitik der Bundesregierung während der Corona-Krise aus einer klassenanalytischen Perspektive. Er zeigt, dass die wirtschaftspolitischen Maßnahmen vor allem denjenigen zugutekommen, die im großen Umfang über Produktionsmittel verfügen. Zwar wurden, etwa durch die Ausweitung des Kurzarbeitergelds, auch die Lohnabhängigen unterstützt. Die dadurch bedingte Entschärfung von Verteilungskonflikten dürfte jedoch um den Preis ihrer künftigen Verschärfung erkauft worden sein. Außerhalb des Schwerpunkts analysieren Tobias Haas und Mariana Schütt die mobilitätspolitischen Aspekte der Corona-Krisenpolitik. Während die Hilfen für die Bahn weit hinter dem Investitionsbedarf zurückbleiben und die Beteiligung des Bundes an der Lufthansa nicht zur ökologischen Neuausrichtung des Unternehmens genutzt wurde, erfreut sich die Autoindustrie einer starken staatlichen Unterstützung. Zwar bekam sie nicht die erwünschte Kaufprämie für Autos mit Verbrennungsmotor, aber der Übergang zur Elektromobilität wird trotz seiner sozialökologischen Fragwürdigkeit großzügig subventioniert.

Michael Brie würdigt Friedrich Engels, dessen Geburtstag sich am 28. November zum 200. Mal jährt. Konkret widmet er sich Engels' 1845 erschienener Schrift über die Lage der arbeitenden Klasse in
England. Diese ist nicht nur eine ebenso materialreiche wie scharfsinnige Beobachtung der miserablen Verhältnisse, unter denen die Lohnabhängigen in den englischen Industriestädten des 19. Jahrhunderts lebten und arbeiteten. Sie schärft vielmehr auch den Blick für die Klassendimension heutiger Krisen, allen voran der Corona-Pandemie.

\section{Literatur}

Dörre, Klaus (2019): Umkämpfte Globalisierung und soziale Klassen. In:Candeias, Mario / Dörre, Klaus / Goes, Thomas E.: Demobilisierte Klassengesellschaft und Potenziale verbindender Klassenpolitik. Beiträge zur Klassenanalyse (2). Berlin: 11-56.

Dowling, Emma / van Dyk, Silke / Graefe, Stefanie (2017): Rückkehr des Hauptwiderspruchs? Anmerkungen zur aktuellen Debatte um den Erfolg der Neuen Rechten und das Versagen der »Identitätspolitik«. In: PROKLA 188 47(3): 411-420. DOI: https:// doi.org/10.32387/prokla.v47i188.69.

Eribon, Didier (2016): Rückkehr nach Reims. Berlin.

Friedrich, Sebastian (2018): Neue Klassenpolitik. Linke Strategien gegen Rechtsruck und Neoliberalismus. Berlin.

Goodhart, David (2017): The Road to Somewhere: The Populist Revolt and the Future of Politics. London.

Graf,Jakob (2019): Kämpferischer Kommunitarismus? Warum wir auch in Zeiten der demobilisierten Klassengesellschaft an einem sozioökonomischen Klassenbegriff festhalten sollten. In: Vester, Michael u.a. (2019): Klassen, Fraktionen, Milieus. Beiträge zur Klassenanalyse I. Manuskripte Neue Folge. Berlin: 89-103.

Kadritzke, Ulf (2016): Zur Mitte drängt sich alles (Teil 2). Die Gegenwart im Lichte historischer Klassenstudien. In: PROKLA 185 46(4): 639-659. DOI: https://doi. org/10.32387/prokla.v46i185.136.

LuXemburg (2017): Neue Klassenpolitik. URL: https://www.zeitschrift-luxemburg.de/, Zugriff: 28.7.2020. 
Merkel, Wolfgang (2017): Kosmopolitismus versus Kommunitarismus: Ein neuer Konflikt in der Demokratie. In: Harfst, Phillip u.a. (Hg.): Parties, Governments and Elites. The Comparative Study of Democracy. Berlin: 9-23.

Milios, Jannis / Economakis, Georg (2014): Mittelklassen, Klassenstellung und politische Klassenpositionen. In: PROKLA 176 44(3): 403-423. DOI: https://doi.org/10.32387/ prokla.v44i176.164.

Nachtwey, Oliver (2016): Die Abstiegsgesellschaft. Über das Aufbegehren in der regressiven Moderne. Berlin

PROKLA-Redaktion (1971): Editorial. In: Probleme des Klassenkampfs 11(1): 1-4.DOI: https://doi.org/10.32387/prokla.v1i1.1224.

- (1980): Editorial.Ökologiebewegung und Arbeiterbewegung - ein Widerspruch? In: PROKLA 39 10(2): 1-14. DOI: https://doi. org/10.32387/prokla.v10i39.1594.

- (1981):Editorial.Probleme mit demKlassenkampf-Marxistische Theorie und soziale Bewegungen.In:PROKLA 4311(2):1-3.DOI: https://doi.org/10.32387/prokla.v11i43.1551. Redaktionskollektiv Gewerkschaften (1975): Kapitalistische Krise, Arbeitslosigkeit und Krise der Gewerkschaftspolitik in der Bundesrepublik. In:Probleme des Klassenkampfs 19/20/215(2):3-8.DOI:https://doi. org/10.32387/prokla.v5i19/20/21.1738.

Reckwitz, Andreas (2019): Die Gesellschaft der Singularitäten. Zum Strukturwandel der Moderne. Berlin.

Riexinger, Bernd (2018): Neue Klassenpolitik. Solidarität der Vielen statt Herrschaft der Wenigen. Hamburg.

Sablowski, Thomas / Thien, Hans-Günter (2018). Die AfD, die ArbeiterInnenklasse und die Linke - kein Problem? In: PROKLA. 190 48(1): 55-72. DOI: https://doi. org/10.32387/prokla.v48i190.32.

Thien, Hans-Günter (2014). Klassentheorien Die letzten 50 Jahre. In: PROKLA 175 44(2): 163-190. DOI: https://doi.org/10.32387/ prokla.v44i175.169.

Wright, Erik Olin (1985): Wo liegt die Mittelklasse? In: PROKLA 58 15(1): 35-62. DOI: https://doi.org/10.32387/prokla. v15i58.1154.

Zeuner, Bodo (1995): »Probleme des Klassenkampfs« im Betrieb. In: PROKLA 100 25(3): 341-379. DOI: https://doi.org/10.32387/ prokla.v25i100.950.

\section{Der PROKLA Förderverein}

Die PROKLA erscheint seit 1971 und bietet politisch engagierte sozialwissenschaftliche und ökonomische Analysen. Allein von den Verkaufserlösen kann sich die PROKLA nicht finanzieren, und in die Abhängigkeit von Parteien oder großen Verlagen wollte sie sich nie begeben. Deshalb wird die PROKLA von einem Förderverein herausgegeben, der »Vereinigung zur Kritik der politischen Ökonomie e.V.«, die jährlich in ihrer Vollversammlung die Redaktion der Zeitschrift wählt und die nächsten Themenschwerpunkte diskutiert.

Kritische Sozialwissenschaft kann nicht dem Markt überlassen werden. Ohne solidarische Strukturen und finanzielle Unterstützung sind Zeitschriften wie die PROKLA kaum möglich. Die finanziellen Beiträge der Vereinsmitglieder ermöglichen das Erscheinen der PROKLA, sie schaffen die Voraussetzungen für Kontinuität und Planbarkeit, wie sie für die Redaktionsarbeit unabdingbar sind. Wir freuen uns über weitere Mitglieder, regelmäßige Spenden oder einmalige Zuwendungen. Weitere Informationen teilen wir gerne per E-Mail mit (redaktion@prokla.de).

Vereinigung zur Kritik der politischen Ökonomie e.V. | Postbank Berlin | IBAN: DE17 100100100538135100 | BIC: PBNKDEFF 